\title{
Germanica
}

\section{Dilettantentum als Kunst: der Fall Thomas Mann}

Le dilettantisme en tant qu'art: le cas Thomas Mann

Dilettantism as art: the case of Thomas Mann

\section{Elena Alessiato}

\section{OpenEdition}

\section{Journals}

Édition électronique

URL : http://journals.openedition.org/germanica/3836

DOI : 10.4000/germanica.3836

ISSN : 2107-0784

\section{Éditeur}

Université de Lille

\section{Édition imprimée}

Date de publication : 30 juin 2017

Pagination : 181-195

ISBN : 9782913857391

ISSN : 0984-2632

\section{Référence électronique}

Elena Alessiato, „Dilettantentum als Kunst: der Fall Thomas Mann“, Germanica [Online], 60 | 2017.

Online erschienen am: 30 Juni 2019, abgerufen am 08 Januar 2021. URL: http://

journals.openedition.org/germanica/3836 ; DOl: https://doi.org/10.4000/germanica.3836 


\title{
Dilettantentum als Kunst: der Fall Thomas Mann
}

\author{
Elena ALESSIATO \\ Universität Turin
}

Am 23.4.1925 schrieb Thomas Mann an Julius Bab: „Dass das Soziale meine schwache Seite ist - ich bin mir dessen voll bewußt und weiß auch, daß ich mich damit in einem gewissen Widerspruch zu meiner Kunstform selbst, dem Roman befinde, der das Soziale fordert und mit sich bringt. Aber der Reiz - ich drücke es ganz frivol aus - des Individuellen, Metaphysischen ist für mich nun einmal unvergleichlich größer"1. Diese Bemerkung macht Thomas Mann in Bezug auf den Zauberberg, sie könnte aber auch für andere Werke von ihm gelten. Denn was er mit seinen Romanen meisterhaft geleistet hat, sind imposante literarische Fresken: große literarische Darstellungen von Zeitepochen, in dem der soziale und historische Kontext als zugleich repräsentativer Hintergrund und symbolische Projektion einer bestimmten Lebenssituation gilt. Das gilt sowohl für den Zauberberg als auch für Doktor Faustus, doch in erheblichem Maß für die Buddenbrooks, den „Roman des Fünfundzwanzigjährigen, an der Schwelle des Jahrhunderts entstanden“. Er, so kommentiert Mann, ,,war ein Werk ganz ohne jenen "Geist im Dienste der Wünschbarkeit", ganz ohne sozialen "Willen",

1. - Brief an Julius Bab (23.4.1925), zit. in: Hans Wysling, „Einführung“ zu Thomas Mann - Heinrich Mann, Briefwechsel 1900-1949, hrsg. v. H. Wysling, Frankfurt a.M., Fischer, 1995, S. 7-54, hier S. 37. 
ganz unpathetisch, unrednerisch, unsentimental, vielmehr pessimistisch, humoristisch und fatalistisch, wahrhaftig in seiner melancholischen Unterwürfigkeit als Studie des Verfalls“2.

Tatsächlich hat Thomas Mann ein ,sozialkritisches Buch`im strengen Sinn des Wortes nie geschrieben. Der Grund dafür liegt maßgeblich in der Tatsache, dass Thomas Manns größtes Interesse sich nicht auf den Fortschritt der Gesellschaft, sondern auf die Entfaltung des Menschen bzw. des Menschlichen richtet. Bereits sehr früh war Thomas Mann sich dessen bewusst, auch weil ein gegensätzlicher Vergleichsmaßstab ihm ganz nah stand. In einem Brief vom 8.11.1913 gesteht er dem vier Jahre älteren Bruder Heinrich ,die Unfähigkeit, mich geistig und politisch eigentlich zu orientieren, wie Du es gekonnt hast“; denn ,eine wachsende Sympathie mit dem Tode“ sei ihm ,tief eingeboren: mein ganzes Interesse galt immer dem Verfall, und das ist es wohl eigentlich, was mich hindert, mich für Fortschritt zu interessieren“3. Um einen grausam zynischen sozialkritischen Roman wie Der Untertan schreiben zu können, brauchte man tatsächlich eine geradlinige politische Orientierung und den politischen Willen, mit einer ganzen gesellschaftlichen Struktur und der entsprechenden Denkweise ins Gericht zu gehen. Vor allem brauchte man die Kraft zu hoffen, dass dadurch auch eine bessere Gesellschaft möglich werden konnte. Nicht oft hat der junge Schriftsteller Thomas Mann solche Kraft in sich gespürt, denn in dem schon zitierten Brief bemerkt er: „Es ist schlimm, wenn die ganze Misere der Zeit und des Vaterlandes auf einem liegt, ohne dass man die Kräfte hat, sie zu gestalten“. Die Willensschwachheit wird fast zur Reue und Lebensverweigerung: „Ich bin ausgedient, glaube ich, und hätte wahrscheinlich nie Schriftsteller werden dürfen“44, stellt Mann fest.

Eigentlich wäre es unzutreffend, Thomas Mann nur als Schriftsteller zu bezeichnen: der Schriftsteller Thomas Mann hat tatsächlich kein sozialkritisches Buch geschrieben. Wenn er trotzdem Sozialkritik ausgeübt hat, so bleibt diese fragwürdig. Sicher ist dagegen, dass Kritik einen wesentlichen Bestandteil seiner künstlerischen Natur und Wirkung ausmacht. Die Kunst Thomas Manns ist insofern kritischer Art, als „seine Anlagen und Bildungsüberlieferungen - moralisch-metaphysischer, nicht politisch gesellschaftlicher Art waren“5. Thomas Mann ist insofern Kritiker, als er Künstler ist. Aber was für eine Kritik übt er aus?

2. - Thomas Mann, Betrachtungen eines Unpolitischen, in: Ders., Große kommentierte Frankfurter Ausgabe (ab jetzt GkFA), Bd. 13, 1, hrsg. von H. Kurzke, Frankfurt a.M., Fischer, 2009, S. 27. Die Betrachtungen eines Unpolitischen werden im Folgenden abgekürzt durch $B e U$, mit Seitenangaben im Text.

3. - T. Mann, H. Mann, Briefwechsel 1900-1949, a.a.O., S. 166.

4. - Ebd.

5. - Thomas Mann, Lebensabriss (1930), in: Ders., Gesammelte Werke in zwölf Bänden (ab jetzt $G W$ ), Bd. XI, Frankfurt a.M., Fischer, 1974, S. 98-144, hier S. 127. Vgl. 


\section{Der Künstler als Kritiker}

Die Suche nach einer Antwort kann mit folgendem Zitat beginnen:

Der Ästhet als Kritiker - und warum sollte nicht Kritik auch ihm Bedürfnis und beinahe Sinn der Sprache sein? - ist wenig geneigt, das Nationale, das Politisch-Soziale als Mittel zwischen Ich und Menschheit ins Auge zu fassen. Seine Kritik ist ebenfalls Selbstkritik, aber nicht im politischen, sondern im moralischen Sinn; sie ist nach innen gerichtet, doch nicht ins politisch Innere der Nation, sondern ins eigene Individuelle, und indem sie das Ich trifft, trifft sie ganz unmittelbar das Menschliche überhaupt. [...] So beruht die Anmaßung des Ästheten darin, dass er das eigene Ich als Träger des AllgemeinMenschlichen insinuiert und das Menschentum nur auf dem Wege über sein Ich sowohl liebt als hechelt, sowohl bejaht als verneint. (BeU, S. 323f.)

Das Zitat umfasst viele Elemente, die Manns Auffassung der Kritik charakterisieren. Ich möchte mich nun auf die Fokussierung von drei Elementen beschränken, deren Verbindung ich im Verlauf des Beitrages klar machen möchte: ich beziehe mich auf den Träger der Kritik, den Gegenstand der Kritik und den Ort der Kritik, d.h. den Text, dem das Zitat entnommen ist.

Denn der Literat, der Schriftsteller, der Journalist oder der Soziologe können nach Thomas Mann keinen Alleinanspruch auf die Darstellung und Ausübung der Kritik erheben. Kritiker kann auch derjenige sein, der das künstlerische Tun nicht zum Handeln verurteilt, sondern zum Wirken bestimmt sieht und der das literarisierte Wort nicht primär oder exklusiv als analytisches Mittel zur erbarmungslosen Zergliederung der Wirklichkeit oder, ganz im Gegenteil, zu deren seelenlosen Nachbildung anwendet. Nach Manns Auffassung - und obgleich es schwierig ist, eine eindeutige und für jede Phase seiner Produktion gültige Definition von Kunst zu finden - ist die Kunst „Leben im Licht des Gedankens“ (BeU, S. 447): das nicht-ästhetisierte künstlerische Wort soll Analyse und Leidenschaft, Kritik und Dichtung, Lebenszerstörung und Lebensfeier, Aufklärung und Verklärung zur Synthese bringen. „Meine Muse ist keine riesige Maid, die zürnend dreinschlägt, sondern ein liebliches

auch die Art und Weise, wie Mann den Künstler beschreibt: „Der Künstler, der doch seiner Natur nach in mindestens so hohem Grad auf Erhaltung wie auf Vernichtung bedacht, auf Glauben, Form, Kultur, Ordnung mindestens so angewiesen ist wie auf Erkenntnis, Kritik und Auflösung, der, mit einem Worte, dem Leben sich mindestens so verbunden fühlen muss wie dem Nichts", in: Thomas Mann, Brief an Hermann Graf Keyserling (1920), in: GW, Bd. XII, a.a.O., S. 593-603, hier S. 600. 
Mägdelein, das Kränze windet und idyllisch singt“" , schrieb Mann schon 1895.

Das mit künstlerischem Blick und dichterischer Feinfühligkeit ausgestattete Ich ist das Subjekt und der spielerische Träger der Kritik, da er in sich selbst das Material für die Ausführung seiner Tätigkeit findet. Die Gleichsetzung von Kritik und Selbstkritik, die das Zitat verdeutlicht, findet ihren Grund darin, dass laut Mann ,Schriftstellertum selbst [...] als ein Erzeugnis und Ausdruck der Problematik, des Da und Dort, des Ja und Nein, der zwei Seelen in einer Brust“ erschien. ,Wozu, woher überhaupt Schriftstellertum, wenn es nicht geistig-sittliche Bemühung ist um ein problematisches Ich?" (BeU, S. 23), fragt sich Mann.

Das Ich schaut sich an und findet dort „einen schlimmen Reichtum an inneren Konflikten, Gegensätzen und Widersprüchen“ (ebd.). Nicht nur die Vielfältigkeit der moralischen Spannungen, sondern auch das dem moralischen Subjekt innewohnende Gewirr von emotionaler Doppeldeutigkeit und intellektueller Klarheit, von Herzensbedürfnis und Realitätssinn gibt Anlass zu einer Kritik, die zugleich als Selbstkampf und Selbstforschung, als Selbsterkenntnis und Selbstüberwindung wirkt. Darin besteht der Gegenstand der Kritik. Bedeutsam ist in diesem Zusammenhang eine Notiz aus der geplanten kunsttheoretischen Abhandlung über „Geist und Kunst“ (1909-1913, zum Großteil 1909-1910): „Manches im Grunde Selbstkritik. Viele der kritisierten Tendenzen auch in mir. Damit, dass ich sie klarstelle, verneine ich sie noch nicht. Eine gewisse psychologische Bosheit gegen das Unbewußte, Naive. Sagen was ist. Gegen sich selbst sprechen, polemisieren. Gewohnt aus der Polemik zu lernen. Polemik und Liebe "7.

Die innerliche Welt des Einzelnen wird als Resonanzraum deräußeren Lebensumstände und -bestimmungen gesehen, doch auf eine solche Art und Weise, dass der Einzelne selbst zum Heraufbeschwörer einer neuen Lebenswelt wird. „Beseelung ist es, welche den Dichter macht“, und Beseelung ist „,nicht die Gabe der Erfindung“, spezifiziert Thomas Mann in dem Essay Bilse und Ich (1906) ${ }^{8}$. Sie ist dagegen die Fähigkeit, „das Abbilden einer Wirklichkeit subjektiv zu vertiefen“, so dass „das Dargestellte nicht mehr ein Abbild jener äußeren Wirklichkeit, sondern eine subjektive Reflexion auf das eigene Ich unter Zuhilfenahme der

6. - Brief an Otto Grautoff (5.3.1895), zit. in: Harald Höbusch, Thomas Mann. Kunst, Kritik, Politik 1893-1913, Tübingen, Basel, Francke, 2000, S. 40.

7. - Thomas Mann, "Geist und Kunst". Thomas Manns Notizen zu einem "Literatur-Essay", in: H. Wysling, P. Scherrer (Hrsg.), Quellenkritische Studien zum Werk Thomas Manns (Thomas-Mann-Studien, Bd. 1), Bern, München, Francke, 1967, S. 123-233, hier S. 162.

8. - Thomas Mann, Bilse und ich, in: GKFA, Bd. 14.1: Essays I 1893-1914, hrsg. v. H. Detering unter Mitarbeit von S. Stachorski, S. 95-111, hier S. 100-101. 
äußeren Realität" ist ${ }^{9}$. Aus dieser Perspektive wird die Wirklichkeit zu einer Quelle ,sinnlicher Mittel zur Darstellung eines Erlebnisses, einer Idee“. Dieselbe Verbindung von Erleben und Wirklichkeit, Idee und Leben wird nach Jahren dadurch neu moduliert, dass Thomas Mann die Fähigkeit, ,aus historischem Detail Ideen sprechen zu lassen und ihm nur dadurch dramatische Spannkraft zu geben“ (BeU, S. 447), als eine der beachtlichsten Tugenden eines Kunstwerkes lobt.

Nun zur Herkunft des Zitats, d. h. dem Werk Thomas Manns, das seit je am problematischsten ist und problematisch diskutiert wurde; das kein Roman ist, von dem Mann sich jedoch wünscht, es werde „als Roman“ gelesen. Ein Werk, das Mann ,als Vorstellung eines bewußt erlebten und dabei auch schon innerlich distanzierten geistigen Schicksals"10 bezeichnet; das nicht als Literatur im engeren Sinn zu betrachten ist, sondern als Ausbund an Kritik und Kritiken: denn die Betrachtungen eines Unpolitischen sind ein polemischer Kriegsessay, der es auf eine chaotische, doch anreizende Weise schafft, ein grandioses Kulturfresko zu liefern und zugleich eine virulente Kulturkritik zu inszenieren.

Die Betrachtungen entsprechen auf eine hoch repräsentative und symptomatische Weise dem Produkt einer ,geistig-sittlichen Bemühung um ein problematisches Ich": dort unternimmt der Autor einen Kampf gegen die eigene Zeit und gegen die zeitbeherrschenden Denkströmungen und Lebenstendenzen, die er im Kontrast zu seinen eigenen Lebensvorstellungen und -wahrnehmungen sieht und fühlt. Zur gleichen Zeit kämpft er mit sich selbst bzw. gegen diejenigen Zeitstimmungen und -bestimmungen, die wider seine Natur sind, doch ihm und seiner schriftstellerischen Wirkung innewohnen. In einem Brief an Hans Korte vom 3. April 1918 kündigt er harmlos an: Dieses ,neue, recht umfangreich[e] Buch [...] enthält allerlei Zeit- und Selbstkritik"“11.

Die Betrachtungen gelten als Versuch, durch kulturell-geistige, sogar religiöse und metaphysische Motivationen die Politik des damaligen deutschen Reiches zu erklären und zu rechtfertigen. Sie stellen das große Fresko der konservativen deutschen Kultur der Zeit dar und gelten als beispielhafte Darstellung der Reaktion des deutschen Kulturbürgertums, das am stärksten in der Tradition verankert war und zu dem auch Thomas Mann gehörte, gegen die Stimmen, die die Modernisierung Deutschlands forderten.

Eigentlich sind solche Inhalte und Denkzusammenhänge für die damalige Kriegspublizistik nicht ungewöhnlich. Was jedoch für die

9. - H. Höbusch, Thomas Mann..., a.a.O., S. 87.

10. - Thomas Mann, Brief an Philipp Witkop (13.9.1918), zit. in: H. Wysling (Hrsg.), Thomas Mann, Teil I: 1889-1917, Passau, Frankfurt a.M., Heimeran/Fischer, 1975, S. 667.

11. - Ebd., S. 661. 
Betrachtungen typisch und besonders bemerkenswert ist, ist die Art und Weise, in der die Argumentation geführt wird: intuitiv und unsystematisch, künstlerisch und synkretistisch, polemisch und eklektisch. Die Betrachtungen sind das Werk eines starken, doch an sich zweifelnden Ichs, das seine eigene Darstellung bzw. Vorstellung der historischen und geistigen Wirklichkeit seiner Zeit dadurch wiedergibt, dass die verschiedenen Denkmotive nach einer assoziativen Logik subjektiv kombiniert und argumentativ variiert werden. Die subjektive, dichterische Komponente der Betrachtungen ist so stark ausgeprägt, dass die Definition besonders zutreffend erscheint, mit der Mann sein Werk bezeichnete: „Künstlerwerk, Künstlerschrift“, „kein Kunstwerk, ja; denn es entstammt einem in seinen Grundfesten erschütterten, in seiner Lebenswürde gefährdeten und in Frage gestellten Künstlertum, einem krisenhaft verstörten Zustande dieses Künstlertums“" (BeU, S. 14).

Interessant ist hier nicht nur zu bemerken, dass Mann die Tätigkeit des Künstlers mit der Krisis seiner künstlerischen Welt in Verbindung setzt, sondern auch, dass solche Krisis Reflex und Folge einer allgemeineren, breiteren Krisis ist, die die ganze Zeitepoche erschüttert und erregt: ,Denn so war die Zeit geartet, dass kein Unterschied mehr kenntlich war zwischen dem, was den einzelnen anging und nicht anging; alles war aufgeregt, aufgewühlt, die Probleme brausten ineinander und waren nicht mehr zu trennen" (BeU, S. 18). Vor eine solche schwankende und verworrene Lage stellte sich Thomas Mann mit der Haltung eines Künstlers. Doch welchen Künstlers?

\section{Der Künstler als Dilettant}

Zahlreich sind die Künstlerbilder, die sich in den Betrachtungen profilieren: der bürgerliche Künstler; der Ethiker; der Pseudokünstler, der dem ästhetisierten Politiker entspricht; der Lyriker; der naive Taugenichts. Der Künstlertypus, auf den ich mich beziehen möchte, ist derjenige, der paradoxerweise in den ersten Seiten des langen Buchkapitels über die „Politik“ porträtiert wird.

Schiller, Flaubert, Tolstoi, Shakespeare und Goethe stellen die besten Beispiele eines Künstlers dar, dessen Haupteigenschaft darin besteht, die Lebenssituationen in der Vielfältigkeit ihrer verschiedenen Aspekte darzustellen, ohne einen Gesichtspunkt zu bevorzugen und ohne die ästhetische Darstellung mit äußerlichen Kriterien zu beladen. Aus ästhetischer Perspektive betrachtet sind alle Aspekte gleich berechtigt. Kraft einer solchen Geistesverfassung kann der Künstler mit kalibrierter Objektivität, mit gleichem Genuss und gleicher Empathie von Krieg und Frieden, von Liebe und Hass, von Gutem und Bösem, von Moralischem und Teuflischem sprechen, und wird jedes Mal der Wirklichkeit gerecht. Unter Anlehnung an einen Ausdruck von August Strindberg bezeich- 
nete Thomas Mann die „Augenverfassung“ des Künstlers als „stereoskopisch“: damit meint er die Fähigkeit, eine globale, gehobene, mehrdimensionale und allumfassende Perspektive auf die Welt und auf die Wirklichkeit einzunehmen. Die Kunst wird hier zum Mittel, um eine solche Mehrdimensionalität nicht nur wiederzugeben, widerzuspiegeln und auszudrücken, sondern auch kompositorisch zu gestalten. Dadurch wird ,die köstliche Überlegenheit der Kunst über das bloß Intellektuelle in ihrer lebendigen Vieldeutigkeit, ihrer tiefen Unverbindlichkeit, ihrer geistigen Freiheit" (BeU, S. 250) geltend gemacht. „Schmarotzerhaft"“ und mit ,dilettierend[er] Einfühlsamkeit“ muss der Künstler handeln. Dadurch, dass er mit Erlebnissen und widerstreitenden Gefühlen, Gestalten und sinnlichem Drang, Geist und Natur, dem Heiligen und dem Profanen experimentiert, bis er diejenige Lebensform entdeckt, die echtes Kunstwerk ist, behauptet und bestätigt der Künstler sein Recht, „mit Gedanken zu spielen, mit Standpunkten Versuche anzustellen, aber ohne sich an etwas zu binden, denn Freiheit ist die Lebensluft des Dichters" (BeU, S. 245, 249) ${ }^{12}$.

Darin besteht die chamäleonhafte Freiheit des Künstlers: in der Veranlagung, ,jedes Ding in das beste Licht [zu stellen] und mit sorgsamem Auge um dasselbe herum[zugehen]“, so dass „man bei der Betrachtung eines Dinges es vor allem für sich gelten [läßt] und sich vor aller Vergewaltigung hüten [muss]" (BeU, S. 544). Gegen die Gewalt des definitorischen Denkens setzt der Künstler die Fähigkeit, ,sich in das Wesen des Friedens und das des Krieges mit der gleichen dilettierenden Einfühlsamkeit, Liebe und freien Anschauung zu vertiefen“. Um eine solche Fähigkeit zu bezeichnen, fügt Mann in den Betrachtungen hinzu: „Das eben war Ästhetizismus, es war die Gesinnungslosigkeit des - ich spreche es aus: des Schmarotzers“ (BeU, S. 245). Was Mann für den unpolitischen Künstler beansprucht, ist die Freiheit des Dilettanten ${ }^{13}$.

Dilettantentum ist eine Kategorie, die typisch für die Kultur der Jahrhundertwende ist und die in Verbindung mit dem breiteren Kulturphänomen der décadence ${ }^{14}$ steht. Die klassische Quelle für die

12. - Schon in einem Beitrag aus dem Jahr 1913 konfrontiert Mann den Künstler mit dem Literaten. So beschreibt er den Künstler als ,,sittlich indifferent, unverantwortlich und unschuldig wie die Natur, deren rechter Sohn er ist. [...] Ein Bursche, der lebt und leben läßt, sinnlich, kindisch, auf Spiel [...]. Man kennt ihn als Freudenmeister an den Höfen der Großen, als unbekümmerten Mitesser am Tisch des reichen Halunken - und kurz, wenn irgendein löblicher Charakterzug diesem sympathischen Gesellen mangeln sollte, so möchte es etwa die Anständigkeit sein“" Thomas Mann, Der Literat, in: GKFA, Bd. 14.1, a.a.O., S. 354-362, hier S. 360.

13. - Über Bedeutung und Funktionen des Dilettantismus bei Mann vgl. Paolo Panizzo, Ästhetizismus und Demagogie. Der Dilettant in Thomas Manns Frühwerk, Würzburg, Könighausen \& Neumann, 2007.

14. - Für eine bündige Einleitung zur Bedeutung des Begriffs vgl. Roger Bauer, 
Bezeichnung dieses Phänomens ist Nietzsche, der im Fall Wagner schreibt:

Womit kennzeichnet sich jede literarische décadence? Damit, dass das Leben nicht mehr im Ganzen wohnt. [...] Das Leben, die gleiche Lebendigkeit, die Vibration und Exuberanz des Lebens in die kleinsten Gebilde zurückgedrängt, der Rest arm am Leben. Überall Lähmung, Mühsal, Erstarrung oder Feindschaft und Chaos: [...] Das Ganze lebt überhaupt nicht mehr: es ist zusammengesetzt, gerechnet, künstlich, ein Artefakt ${ }^{15}$.

Prototyp des Dilettanten ist dann Wagner als derjenige Künstler, der sich der künstlerischen bzw. künstlichen Mittel bedient, um ein Ganzes heraufzubeschwören, das erst die Illusion der Lebendigkeit bzw. des echten Lebens erzeugt, dessen eigentliches Ziel aber darin liegt, eine bombastische Wirkung auf das Publikum bzw. auf die Masse auszuüben. Wagner war insofern moderner Künstler und „Kind seiner Zeit“, der Zeit der Décadence, als er ,der Meister des ganz Kleinen“ war ${ }^{16 .}$

Für beide Begriffe, Dilettantismus und Décadence, ist jedoch Frankreich das Herkunftsland: die Namen von Charles Baudelaire und Théophile Gautier sind hier zu nennen. Für den Einfluss auf die deutschen Autoren - auf Nietzsche und durch ihn dann auf Heinrich und Thomas Mann - sind aber die Schriften von Paul Bourget besonders wichtig. Gerade bei Bourget treten Begriffe wie décomposition, Fragmentierung, Zerstückelung und Zersetzung hervor, um einen Zustand der Gesellschaft zu fokussieren, in der die Wahrnehmung des Ganzen durch das Überhandnehmen der einzelnen Teile verloren gegangen ist ${ }^{17}$. Als Spur ex negativo von diesem verloren gegangenen Ganzen wirkt die Literatur: einerseits gilt sie als Ausdruck eines orgiastischen Individualismus, der die ganzen Energien partikularistisch und narzisstisch verschlingt; andererseits wird sie als Lebenssensorium geltend gemacht, das die zeitspezifischen Seelenzustände registriert, darunter auch die Mischung aus Unsicherheit, Lebenslähmung, Naivität

„"Décadence": histoire d'un mot et d'une idée“, Cahiers romains d'études littéraires, I (1978), S. 55-71. Über Manns Verständnis der décadence vgl. T. Mann, „Meine Zeit“ (1950), in: GW, Bd. XI, a.a.O., S. 302-324, hier S. 311.

15. - Friedrich Nietzsche, Der Fall Wagner, in: Ders., Kritische Studienausgabe, hrsg. v. G. Colli u. M. Montinari, Bd. 6, Berlin, New York, de Gruyter, 1988², S. 9-53, hier S. 27-28, zit. in H. Höbusch, Thomas Mann, a.a.O., S. 121.

16. - F. Nietzsche, Nietzsche contra Wagner und Der Fall Wagner, in: Ders., Kritische Studienausgabe, a.a.O., Bd. 6, jeweils S. 11 und 418, zit. in H. Höbusch, a.a.O., S. 138-139.

17. - Vgl. den Abschnitt über Théorie de la décadence, in: P. Bourget, Essais de Psychologie contemporaine. Études littéraires, Paris, Gallimard, 1993, S. 13-18. 
und Gedankenlosigkeit, die sich aus der Unmöglichkeit ergibt, eine sichere, ganzheitliche und einheitliche Lebensvorstellung zu haben.

In diesem Rahmen findet der Dilettant seinen (T)Raum. In seinen Essais de psychologie contemporaine bezeichnet Bourget den Dilettantismus als ,eine sehr scharfsinnige und gleichzeitig angenehme Veranlagung des Geistes, die wechselnd unser Wohlgefallen an den verschiedenen Äußerungen des Lebens weckt und uns veranlaßt, uns allen diesen Äußerungen vorübergehend anzupassen, ohne uns einer einzigen völlig hinzugeben“ ${ }^{18}$. Die Unfähigkeit zur Selbstbehauptung und die Unfähigkeit des Willens bilden den Kernpunkt einer geistigen Haltung, die, wie Bourget in Cosmopolis schreibt, der Kernpunkt der Décadence ist ${ }^{19}$. Gerade solche Handlungsunfähigkeit stellt jedoch die Voraussetzung für das einzige für den Dilettanten typische Vermögen dar: d. h. die Fähigkeit, sich in die Gedanken und Existenzformen anderer hineinzuversetzen, in ihnen aufzugehen und sich in ihnen zu verlieren. Das Außenseitertum wird durch die Begegnung und produktive Wahrnehmung der Widersprüchlichkeit und Vielstimmigkeit der Wahrheiten abgebüßt. Bourget macht solches Vermögen unter Anlehnung an Renan exemplarisch klar:

Die Ansicht, daß viele einander widersprechende Standpunkte ihre Berechtigung haben, läßt ihn [Renan] nicht los und hindert ihn, die kampfbereite Stellung einzunehmen, welche uns, den Schülern des unzulänglichen Dogmatismus der früheren Zeiten, als die einzige Art erscheint, die Wahrheit zu bekräftigen. Aber gerade das macht aus dem Dilettantismus eine Art neuer Dialektik, dank welcher die Intelligenz an der unendlichen Reichhaltigkeit der Dinge einen Anteil gewinnt ${ }^{20}$.

18. - Zit. in H. D. Heimendahl, Kritik und Verklärung. Studien zur Lebensphilosophie Thomas Manns in den »Betrachtungen eines Unpolitischen", »Der Zauberberg«, »Goethe und Tolstoi « und »Joseph und seine Brïder«, Würzburg, Königshausen \& Neumann, 1998, S. 36: ,[...] une disposition de l'esprit, très intelligente à la fois et très voluptueuse, qui nous incline tour à tour vers les formes diverses de la vie et nous conduit à nous prêter à toutes ces formes sans nous donner à aucune". (P. Bourget, Ernest Renan, in: Ders., Essais de Psychologie contemporaine, a.a.O., S. 36). Hier bezeichnet Bourget den Dilettantismus auch als „science délicate de la métamorphose intellectuelle et sentimentale" (ebd.).

19. - „L'incapacité d'affirmer" und „l'incapacité de vouloir“ sind „le fond du fond de la décadence“: Zit. in R. Bauer, Der Unpolitische und die Décadence, in: H. Gockel, M. Neumann und R. Wimmer (Hrsg.), Wagner - Nietzsche - Thomas Mann. Festschrift für Eckhard Heftrich, Frankfurt a.M., Klostermann, 1993, S. 279-297, hier S. 288. Vgl. P. Bourget, Essais de Psychologie contemporaine, a.a.O., S. 99-100: „L'abondance des points de vue, cette richesse de l'intelligence, est la ruine de la volonté, car elle produit le dilettantisme et l'impuissance énervée d'êtres trop compréhensifs".

20. - Zit. in H.D. Heimendahl, Kritik und Verklärung, a.a.O., S. 37: „La légitimité de beaucoup de points de vue contradictoires l'obsède [Renan] et l'empêche de prendre cette position de combat qui nous paraît la seule façon d'affirmer la vérité, à nous, les disciples de l'insuffisant dogmatisme d'autrefois. Mais c'est précisément ce qui fait du 
Einige Jahre später kehrt Bourget die Vorzeichen seiner Beurteilung um. Die Feinfühligkeit wird nun als Lebensuntauglichkeit entlarvt, der Mangel an festen Wahrheiten wird als Lebensschwäche abgetan und die politische und moralische Standpunktlosigkeit wird mit dem Vorwurf nihilistischen (Un)Glaubens gebrandmarkt ${ }^{21}$. Obwohl die explizit zitierte Quelle eine andere war, scheint diese Sicht in den Seiten der Betrachtungen eines Unpolitischen widerzuhallen, die die stereoskopische Geistesverfassung des Künstlers als eine neue bzw. erneuerte Form des Dilettantentums darstellen:

Wer kann definieren, was Freiheit, was Despotismus, was Zivilisation und was Barbarei ist? Wo sind die Grenzen zwischen diesen Begriffen? Wer hat in seiner Seele einen so unfehlbaren Maßstab für Gut und Böse, daß er mit ihm alle die flüchtigen und verworrenen Tatsachen zu messen vermöchte? Wer hat schon je einen Zustand gesehen, wo Gut und Böse nicht miteinander vermengt wären? (BeU, S. 248)

Auch im Laufe seiner intellektuellen und künstlerischen Entwicklung kehrte Mann Meinungen und Beurteilungen in ihr Gegenteil um: vom Bajazzo zu Tonio Kröger, vom Literaten zum Zivilisationsliteraten. Es ist jedoch unleugbar, dass Mann schon sehr früh für das Thema sensibilisiert war. In einem Brief an Otto Grautoff (Mai 1895) schrieb er: „Du liest eine Dichtung, die Eindruck auf Dich macht, und wenn Du Dich dann hinsetzt um zu schreiben, so fühlst und schreibst Du unwillkürlich in ihrem Stil. Das nennt man eben Dilettantismus; oder es gehört wenigstens dazu“ 22 .

Als Vermittler einer solchen geistig-ästhetischen Einstellung war die Rolle des Wiener Literaturkritikers Hermann Bahr sehr wichtig. Die Widmung von Manns Prosa-Skizze Vision (1893) galt „dem genialen Künstler Hermann Bahr“, und einige Stellen aus dem Briefwechsel mit Otto Grautoff ${ }^{23}$ beweisen Manns Würdigung und Interesse für denjenigen Seelenforscher und Tendenzensucher und -entdecker, der sich als

dilettantisme une sorte de dialectique d'un genre nouveau, grâce à laquelle l'intelligence participe à l'infinie fécondité des choses“" (P. Bourget, Ernest Renan, a.a.O., S. 39).

21. - „Seulement, il n'a jamais cru, il ne croira jamais à aucune, pas plus qu'il ne croira jamais à quoi que ce soit, sinon au jeu amusé de son esprit qu'il a transformé en un outil de perversité élégante. Le bien et le mal, la beauté et la laideur, le vice et la vertu lui paraissent des objets de simple curiosité. [...] Pour lui, rien n'est vrai, rien n'est faux, rien n'est moral, rien n'est immoral“. Zit. aus P. Bourget, Le disciple (1888), in: H. D. Heimendahl, Kritik und Verklärung, a.a.O., S. 38.

22. - T. Mann, Briefe an Otto Grautoff (1894-1910) und Ida Boy-Ed(1903-1928), Frankfurt a.M., Fischer, 1975, S. 51.

23. - Z. B. der Brief vom 5.3.1895: Bahr sei ,l'homme de tête und habe immer die richtigen Instinkte für den letzten und kommenden Zeitgeist“, zit. in R. Bauer, Der Unpolitische und die Décadence, a.a.O., S. 282. 
„ein Enthusiast und Don Juan aller künstlerischen Formen“ betrachtete. Über sich selbst schrieb Bahr: „Ich suche geflissentlich vielmehr das Geringe gern: leise, kleine, kaum vernehmliche Gefühle, schwanke Stimmungen der Nerven, die entwischen, feine, flüchtige und rasche Noten, die verhuschen“24. Noch 1954 erinnert sich Mann des „Wiener Essayisten von beträchtlicher Neugier und Spürnäsigkeit“25. Es ist doch wahr, dass bei Bahr der Dilettant einer scharfsinnigen Kritik ausgesetzt wurde, die innerhalb einer kritischen Durchleuchtung der Moderne funktionalisiert wurde ${ }^{26}$. Zu Recht bemerkt Barbara Beßlich, dass ,die Unterscheidung von ästhetischem Gespür und artistischer Potenz für Bahr den Dilettanten vom Künstler abgrenzte" 27 . Aber gerade die dilettantische Ungebundenheit des Kritikers ist das, was der Schriftsteller Thomas Mann rezeptiv verinnerlicht und durch intellektuelle und kritische Umwege zum künstlerischen Vermögen umwandelt. In Manns Zauberhänden wird die untugendhafte Fähigkeit des Dilettanten zur künstlerischen Tugend: Sie wird zum Künstlertum als Kritik, spezifischer noch, zur Zeit- und Kulturkritik.

\section{Künstlertum als (dilettantische) Kritik}

Die Funktion der literarischen Kritik wurde von Bourget unter dem Hinweis auf die Kategorien von „Sensibilität“, „hellsehendem Mitgefühl“ und „Geschichtsschreibung des sittlichen Lebens“ bestimmt; indem er sich einer physiologischen Terminologie bedient, spricht Bahr dabei von „Nervenkunst“: die Literatur, besonders in ihrem kritischen Bestand, scheint dazu befähigt, die Nerven- und Geisteszustände einer Gesellschaft $\mathrm{zu}$ registrieren und gestalterisch darzustellen. Dilettantenhaft handelt der Kritiker insofern, als er von dem Willen getrieben wird, ,die Fülle der Noten, den Schwall und Strudel ihrer gischenden Flut, ihren bunten Sturm zu formen“. Und dabei fügt Bahr hinzu: ,Nicht eine einzelne reizt mich, sondern das Flirren und Flackern ihrer bewegten Menge nur, wie sie sich berstend streifen, stoßen und reiben; in den Grund will ich keine dringen, aber die ganze Fläche dieser

24. - Hermann Bahr, Bahr über sich selbst, in: Ders., Hermann-Bahr-Buch, Berlin, Fischer, 1913 (Nachdruck Unikum-Verlag, Barsinghausen, 2012), S. 18-23, hier S. 19.

25. - T. Mann, Vorwort zu dem Hörspiel >Königliche Hoheit “ in: GW, Bd. XII, a.a.O., S. 579, zit. in J. Eder, „Allerlei Allotria“. Grundzüge und Quellen der Essayistik bei Thomas Mann, Bonn, Bouvier, 1993, S. 107.

26. - Vgl. H. Bahr, Zur Kritik der Moderne. Gesammelte Aufsätze, Zürich, Verlags-Magazin 1890; Ders., Die Überwindung des Naturalismus. Zweite Folge von «Zur Kritik der Moderne», Dresden, Leipzig, Pierson 1891; Ders., Studien zur Kritik der Moderne, Frankfurt a.M., Rütten \& Loening, 1894.

27. - Barbara Beßlich, Wege in den >Kulturkrieg〔. Zivilisationskritik in Deutschland 1890-1914, Darmstadt, Wissensch. Buchgesellschaft, 2000, S. 129. 
breiten Zeit möchte ich fassen, den vollen Taumel aller Wallungen auf den Nerven und Sinnen. Das ist mein Verhängnis“"28.

Dass das auch sein eigenes Verhängnis sein konnte, hat wahrscheinlich Thomas Mann selbst gespürt, als er 1896 in dem Essay Kritik und Schaffen schrieb: „Der Kritiker [hat] aufgehört, ein Richter zu sein und ist zum Erklärer geworden. Denn [...] ist es des Kritikers Kunst, fremde Persönlichkeiten in sich aufzunehmen, in fremden Persönlichkeiten zu verschwinden, durch sie die Welt zu sehen und aus ihnen heraus ihre Worte, ja das Entstehen ihrer Werke zu erklären. [...] Der Kritiker, dieser Verwandlungskünstler, [ist] der vollendetste Typus des "Dilettanten”“29.

In diesem Sinnzusammenhang wirkt der Kritiker als Psychologe eines ganzen zeitbedingten Geistes- und Lebenszustandes, dessen Kenntnis dem Niveau von Selbsterkenntnis einer Kultur entspricht. Sympathie, Skeptizismus und Genuss sind die drei Komponenten einer solchen Kenntnis, spezifiziert Bourget ${ }^{30}$. Dies sind auch die Eigenschaften, die der junge Thomas Mann mit den Dilettanten verbindet, als er sie 1896 auf folgende Weise beschreibt: „Typus des skeptischen ästhetisierenden Genußmenschen [...], letzte Ausläufer ihrer Rasse [...], die ererbte Kräfte, geistige und materielle, verzehren, ohne sie zu vermehren“ 31 . Und noch gerade in den Betrachtungen gewinnt die Skepsis gegenüber hartnäckigen Meinungen, fest geglaubten Wahrheiten und endgültigen Ansichten eine für das feinfühlige Wahrnehmungsvermögen des Künstlers konstitutive und wesentliche Rolle:

Skepsis ist eine selbst intellektuelle Einstellung gegenüber Intellektuellem, sie ist nicht Anti-Intellektualismus, denn dieser bedeutet Ehrfurcht, und der Begriff der Skepsis ist nie ohne einen Einschlag von

28. - H. Bahr, Bahr über sich selbst, a.a.O., S. 22.

29. - Thomas Mann, Kritik und Schaffen, in: GKFA, Bd. 14.1, a.a.O., S. 47-50, hier S. 48-49.

30. - Zit. in J. Eder, „Allerlei Allotria“..., a.a.O., S. 119: „Es ist in der Tat schwierig, aus sich herauszugehen und sich eine gänzlich andere Art zu leben vorzustellen, noch schwieriger aber ist es, über diese Vorstellungen hinauszugehen und, wenn auch nur für einige Minuten, sich selbst, als wenn man ein anderes Kleid anzieht, [...] in diese Art zu leben zu versetzen. Sympathie würde dafür nicht genügen, vielmehr ist dazu ein verfeinerter Skeptizismus, verbunden mit der Kunst, den Skeptizismus in ein Werkzeug des Genusses zu verwandeln, notwendig“. (P. Bourget, Ernest Renan, in Ders.: Psychologische Abhandlungen über zeitgenössische Schriftsteller, übersetzt von A. Köhler, Minden i.W., Bruns, 1903, S. 52).

31. - Thomas Mann, Ein nationaler Dichter, in: Ders., GKFA, Bd. 14.1, S. 40-43, S. 40. Vgl. dazu, was Hans R. Vaget als gemeinsamen Nenner aller Dilettantismusauffassungen identifiziert: den ,Typ eines problematischen, tastenden Intellektuellen“, dem ,in der Kunst wie in anderen geistigen Bereichen eine Haltung des spielerisch-uneigentlichen Probierens und oberflächlichen Berührens eignet". H. R. Vaget, „,Der Dilettant. Eine Skizze der Wort- und Bedeutungsgeschichte“, Jahrbuch der Schillergesellschaft, 14 (1970), S. 131-158, hier S. 152. 
Frivolität. Ehrfurcht und Zweifel, letzte Gewissenhaftigkeit und letzte Ungebundenheit - gibt es diese Verbindung? Doch, es gibt sie, denn sie macht das Wesen der ästhetizistischen Weltanschauung aus. ( $\mathrm{BeU}$, S. 252)

Etwas weniger verblüffend ist die Gemeinsamkeit solcher Weltanschauung mit derjenigen des ,modernen Dilettanten“, den eine Literaturwissenschaftlerin so bestimmt: „eine Mischung aus Skeptizismus, Unverbindlichkeit, Beobachtungssucht und Hedonismus"32. Modern ist Thomas Mann aber auch, weil er dem Skeptizismus nicht das letzte Wort einräumt. Er schafft dagegen die denkerische Voraussetzung für eine weitere intellektuelle und menschliche Entwicklung: für Emanzipation. Denn Mann sagt von sich selbst:

Ich gehöre geistig jenem über ganz Europa verbreiteten Geschlecht von Schriftstellern an, die, aus der décadence kommend, zu Chronisten und Analytikern der décadence bestellt, gleichzeitig den emanzipatorischen Willen zur Absage an sie - sagen wir pessimistisch: die Velleität dieser Absage im Herzen tragen und mit der Überwindung von Dekadenz und Nihilismus wenigstens experimentieren. (BeU, S. 220)

Dilettantenhaft und ungebunden, polemisch und experimentell, manchmal leichtsinnig argumentiert der unpolitische Autor der nicht-unpolitischen Betrachtungen, um letztendlich eine Lebensund Werteinstellung durchzusetzen und $\mathrm{zu}$ verteidigen, die an sich den Willen ausspricht, den nihilistischen Unglauben, die dekadente Lebensfremdheit und die amoralische Willensschwäche des Lebensdilettanten aufzuhalten und zu schwächen, sogar zu überwinden.

Das Ideal, das als Hebelpunkt für eine solche Überwindung zur Geltung gebracht wird, ist dasjenige der deutschen Kultur, damals betrachtet als die Gesamtheit von Werten, Idealen und historischen Symbolen, die das Wesen der deutschen Seele nach der damaligen kulturbedingten Vorstellung ausmachten. Die Zeitkritik, die in den Betrachtungen mit virulenter Stimme zum Ausdruck kommt, hat zum Ziel, die Tendenzen, die Phänomene, die Lebens- und Gedankenweisen anzuprangern, die für die Aufrechterhaltung und die Entfaltung der deutschen Kultur und des deutschen Geistes als feindlich, fremd und gefährlich wahrgenommen wurden. In den Betrachtungen scheint die Absicht verwirklicht zu werden, die viele Jahre zuvor notiert wurde:

Nichts von brennenderem Interesse, als die Kritik der Modernität: das fühlte ich schon mit neunzehn, als ich zum ersten Mal Nietzsches

32. - Joëlle Stoupy, Maître de l'heure. Die Rezeption Paul Bourgets in der deutschsprachigen Literatur um 1890, Frankfurt a.M., Peter Lang, 1996, S. 42. 
Wagner-Kritik las [...]. Es fehlt in Deutschland an Psychologie, an Erkenntnis, an Reizbarkeit, Gehässigkeit der Erkenntnis, es fehlt an kritischer Leidenschaft... [...]. Der Kampf gegen die Zeit und gegen das, was sie im Triumphe emporhebt, braucht nicht notwendig aus persönlicher Verbissenheit hervorzugehen. Große Beispiele lehren, dass er dem Willen zur Selbsterkenntnis entspringen und die Selbsterkenntnis der Zeit, ihre Selbstüberwindung fördern kann ${ }^{33}$.

Nicht aus Fremdheit und Hass, sondern aus Gemütsnähe und widersprüchlicher Anteilnahme ergibt sich der Wille, sich selbst zu erforschen und die eigene Zeit kritisch zu erleben, indem ihre Vielfältigkeit offenkundig gemacht wird. Erst ein solches, durch die kritische Untersuchung ermöglichtes und wahrgenommenes Zeiterlebnis macht die Überwindung des Krisenzustandes wahrscheinlich. Denn die Zivilisationskritik, die vielen polemischen Seiten der Betrachtungen zugrunde liegt, gilt als eine Übung zur Zivilisationserkenntnis, die darauf zielt, die Kulturkritik als Kulturverständnis möglich und klar $\mathrm{zu}$ machen. Mit Bezug auf den geschichtlichen damaligen Rahmen kann man auch sagen, dass die Zeitkritik, die die Betrachtungen kundgeben, darauf gerichtet ist, die Zivilisation(skritik) zu überwinden, um dadurch dem Ideal der deutschen Kultur Leben, Vollständigkeit und Anerkennung liefern zu können. Der Versuch, die deutsche Kultur zu neuem Leben zu erwecken, bietet die Hoffnung, auf die Orientierungslosigkeit der Jahrhundertwende reagieren und sich der korrumpierenden und lähmenden Lebensschwäche entziehen zu können. Doch gerade jener existenzielle, intellektuelle und geistige Zustand versetzte den Künstler in die Lage, die intellektuellen und emotionalen Mittel zu erobern und zu verinnerlichen, die es ihm dann ermöglichten, tief in den geistigen Strudel der Kriegszeit einzutauchen und ihre vieldeutigen Widersprüchlichkeiten aufzurollen.

Das Ringen um ein problematisches Ich ist nun nicht mehr zur Lebensfremdheit verurteilt, denn der kulturkritische Kampf des Unpolitischen gab solchem Ringen das Ziel und den Sinn, die der ehemalige Dilettant unfähig war, sich allein vorzustellen: Selbsterkenntnis und Selbstüberwindung werden jetzt im Namen eines mächtigen Lebensideals - und zwar des deutschen Lebensideals - als möglich nicht nur gedacht, sondern sogar geglaubt.

„Voll von Abscheu, Widerstand und Furcht": so erscheinen die „goldbraunen, bläulich umschatteten Augen“ des kleinen Johann Buddenbrook dem Leben gegenüber. Solche Augen gelten als zeitbestimmendes Zeichen, denn gerade diejenige ,Widersetzlichkeit, die sensitivsittliche Revolte gegen "das Leben wie es ist", gegen das Gegebene, die 
Wirklichkeit, die "Macht" - diese Widersetzlichkeit [ist das] Merkmal des Verfalls, der biologischen Unzulänglichkeiten" (BeU, S. 28), unter der mehr als eine Generation von europäischen Künstlern, Kritikern und Literaten zu leiden hatte. Unter ihnen auch Thomas Mann, der aber sowohl in der künstlerhaften Ausübung der Kritik seiner eigenen Zeit gegenüber als auch in dem entsprechenden künstlerischen Ab-Bilden eines visionären, seelenerfüllenden, zielgebenden und lebensverklärenden Ideals - desjenigen der deutschen Kultur - das Prinzip und den Grund dazu erkennt, die in sich innewohnenden verfallenden, dekadenten Triebe zu beherrschen und zu überwinden.

Thomas Mann war insofern ein Mensch seiner Zeit, als er in sich selbst Lebenseinstellungen und intellektuelle Veranlagungen fand, die er bei seinem Feind, dem Zivilisationsliteraten, und im Allgemeinen bei seiner zivilisierten bzw. literarisierten und politisierten Zeit verschmähte. In dem Maße, in dem er als Psychologe seiner selbst und seines problematischen Ichs wirkte, schaffte er es, seine eigene Zeit psychologisch zu erkennen und dabei Psychologe seiner eigenen Welt zu sein. Dadurch konnte er mit dem geistigen Zustand einer Kulturwelt - der des protestantischen, kulturbürgerlichen, monarchischen Deutschlands - mitfühlen, die sich als schwankend und ängstlich erlebte und sich den gewaltsamen Angriffen der Modernisierung, des Fortschritts, der westlichen Demokratisierung ausgesetzt sah. Als Sohn jener Welt stellte der literarisch begabte Künstler Thomas Mann psychologisch fundierte Fragen über seine eigene Stellung in einer sich rasch und traumatisch verändernden Welt. Tatsächlich hat der Dilettant kein sozialkritisches Buch geschrieben. Er hat trotzdem seinen Beruf und seinen eigenen kulturlegitimierten Stand in der geschichtlichen und gesellschaftlichen Welt gefunden. Der vergeistigte Krieg gab ihm dafür Anlass und Glaubensinhalt. 
